Check for updates

Cite this: Chem. Commun., 2020,

56,11505

Received 31st July 2020

Accepted 24th August 2020

DOI: $10.1039 / \mathrm{d} 0 \mathrm{cc} 05214 \mathrm{k}$

rsc.li/chemcomm

\section{A large kinetic isotope effect in the reaction of ascorbic acid with 2-phenyl-4,4,5,5- tetramethylimidazoline-1-oxyl 3-oxide (PTIO') in aqueous buffer solutions $\dagger$}

\author{
Ikuo Nakanishi, (D) *a Yoshimi Shoji, ${ }^{a}$ Kei Ohkubo, (D) ab Toshihiko Ozawa, \\ Ken-ichiro Matsumoto (D) ${ }^{a}$ and Shunichi Fukuzumi (iD)*de
}

\begin{abstract}
A large kinetic isotope effect (KIE, $\left.k_{\mathrm{H}} / k_{\mathrm{D}}\right)$ of 12.8 was observed for the hydrogen-transfer reaction from ascorbic acid to 2-phenyl4,4,5,5-tetramethylimidazoline-1-oxyl 3-oxide (PTIO*) in a phosphate buffer solution $(0.05 \mathrm{M}, \mathrm{pH} / \mathrm{pD} 7.0)$ at $298 \mathrm{~K}$. The isotopic difference in the activation energies $\left(6.8 \mathrm{~kJ} \mathrm{~mol}^{-1}\right)$ determined from the temperature dependence of the KIE suggests that quantum mechanical tunneling may partly play a role in the reaction, although the isotopic ratio of the Arrhenius prefactor $\left(A_{\mathrm{H}} / A_{\mathrm{D}}=\right.$ 0.86 ) is within the semiclassical limits.
\end{abstract}

Quantum mechanical tunneling in hydrogen-transfer reactions ${ }^{1-3}$ in biological redox systems has attracted considerable attention with regard to the quantum mechanical behaviour in biology in recent years. ${ }^{4}$ Uršic et al. reported that a large kinetic isotope effect $\left(\mathrm{KIE}, k_{\mathrm{H}} / k_{\mathrm{D}}\right)$ of 24.2 was observed in water for the hydrogentransfer reaction from ascorbic acid $\left(\mathrm{AscH}_{2}\right)$, one of the representative water-soluble antioxidants, to 2,2,6,6-tetramethylpiperidine1-oxyl (TEMPO) radicals. ${ }^{5}$ This is the first report about hydrogen tunneling in a reaction involving $\mathrm{AscH}_{2}$. On the other hand, Li has recently reported a new and simple antioxidant assay in vitro using 2-phenyl-4,4,5,5-tetramethylimidazoline-1-oxyl 3-oxide radicals (PTIO ${ }^{\bullet}$ ), one of the nitronyl nitroxide radicals, where a hydrogen transfer occurred from antioxidants to PTIO ${ }^{6}{ }^{6}$ However, little is known about the kinetics of the reaction between

${ }^{a}$ Quantitative RedOx Sensing Group, Department of Basic Medical Sciences for Radiation Damages, National Institute of Radiological Sciences (NIRS), Quantum Medical Science Directorate, National Institutes for Quantum and Radiological Science and Technology (QST), Inage-ku, Chiba 263-8555, Japan.

E-mail: nakanishi.ikuo@qst.go.jp; Fax: +81-43-255-6819; Tel: +81-43-206-3131

${ }^{b}$ Institute for Advanced Co-Creation Studies, Open and Transdisciplinary Research Initiatives, Osaka University, 2-8 Yamada-oka, Suita, Osaka 565-0871, Japan

${ }^{c}$ Nihon Pharmaceutical University, Kitaadachi-gun, Saitama 362-0806, Japan

${ }^{d}$ Department of Chemistry and Nano Science, Ewha Womans University, Seoul 03760, Korea

${ }^{e}$ Faculty of Science and Engineering, Meijo University, Nagoya, Aichi 468-8502, Japan

$\dagger$ Electronic supplementary information (ESI) available: Experimental details. See DOI: $10.1039 / \mathrm{d} 0 \mathrm{cc} 05214 \mathrm{k}$ antioxidants and $\mathrm{PTIO}^{\bullet}$, as well as the KIE. We report herein the observation of a large kinetic isotope effect for the reaction of $\mathrm{AscH}_{2}$ with $\mathrm{PTIO}^{\bullet}$ in a phosphate buffer. A possibility of the involvement of quantum mechanical tunneling is also discussed based on the temperature dependence of the KIE.

When $\mathrm{AscH}_{2}$ was added to the phosphate buffer solution (0.05 M, pH 7.0) of PTIO $^{\circ}$, the bands at 345 and $560 \mathrm{~nm}$ decreased immediately with clear isosbestic points at 218, 244, 279 and $313 \mathrm{~nm}$ as shown in Fig. 1. Since the $\mathrm{p} K_{\mathrm{a}}$ value of $\mathrm{AscH}_{2}$ is reported to be $4.1,{ }^{7} \mathrm{AscH}_{2}$ undergoes deprotonation and exists in its anionic form, $\mathrm{AscH}^{-}$, in phosphate buffer solution (0.05 M, pH 7.0). Thus, this spectral change indicates that $\mathrm{AscH}^{-}$efficiently scavenged $\mathrm{PTIO}^{\bullet}$ in phosphate buffer. The spectral titration (inset of Fig. 1) shows that the stoichiometry of the reaction is given by eqn (1), where $\mathrm{AscH}^{-}$reacts with $2 \mathrm{PTIO}^{\bullet}$.<smiles></smiles><smiles>CC1(C)N(O)C(c2ccccc2)=[N+]([O-])C1(C)C</smiles>

The decay of the absorbance at $560 \mathrm{~nm}$ monitored by a stopped-flow technique obeyed pseudo-first-order kinetics, when the concentration of $\mathrm{AscH}_{2}\left(\left[\mathrm{AscH}_{2}\right]\right)$ was maintained at more than a 10 -fold excess of $\mathrm{PTIO}^{\bullet}$ concentration (Fig. 2). The pseudo-first-order rate constants $\left(k_{\text {obs }}\right)$ linearly increased with increasing $\left[\mathrm{AscH}_{2}\right]$ (Fig. 3). From the slope of the linear plot, the second-order rate constant $\left(k_{\mathrm{H}}\right)$ for the scavenging reaction of PTIO $^{\bullet}$ by $\mathrm{AscH}_{2}$ [eqn (2)] was determined in a phosphate buffer 


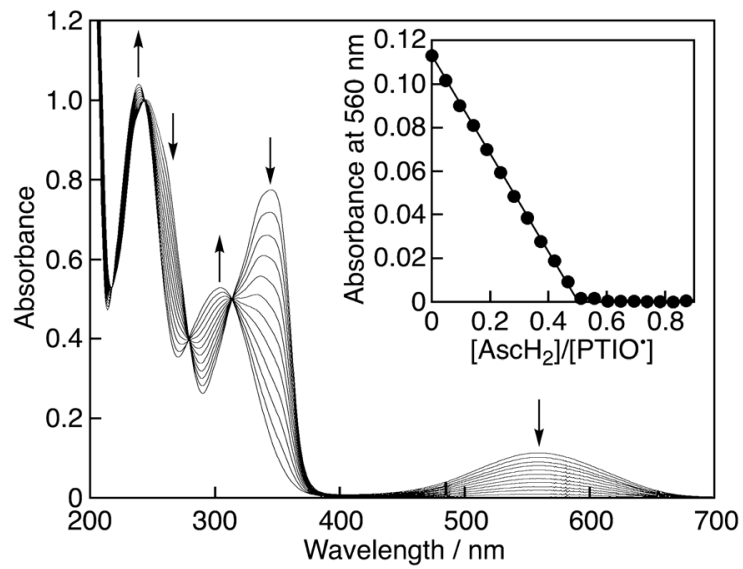

Fig. 1 Spectral change observed upon addition of $\mathrm{AsCH}_{2}(0-5.2 \times$ $10^{-5} \mathrm{M}, 4.6 \times 10^{-6} \mathrm{M}$ each) to $\mathrm{PTIO} \cdot\left(1.0 \times 10^{-4} \mathrm{M}\right)$ in phosphate buffer $(0.05 \mathrm{M}, \mathrm{pH} 7.0)$. Inset: Plot of the absorbance at $560 \mathrm{~nm}$ vs. $\left[\mathrm{AsCH}_{2}\right] /[\mathrm{PTIO} \cdot]$

$(0.05 \mathrm{M}, \mathrm{pH} 7.0)$ to be $2.4 \times 10^{3} \mathrm{M}^{-1} \mathrm{~s}^{-1}$. This value is smaller than that determined for the reaction between $\mathrm{AscH}_{2}$ and $\beta$-cyclodextrin-solubilised 2,2-diphenyl-1-picrylhydrazyl radicals $\left(\mathrm{DPPH}^{\bullet}\right)^{8}$ under the same experimental conditions $\left(k_{\mathrm{H}}=5.6 \times\right.$ $\left.10^{3} \mathrm{M}^{-1} \mathrm{~s}^{-1}\right)$. Thus, the reactivity of PTIO ${ }^{\bullet}$ toward $\mathrm{AscH}_{2}$ is lower than that of $\mathrm{DPPH}^{\bullet}$.

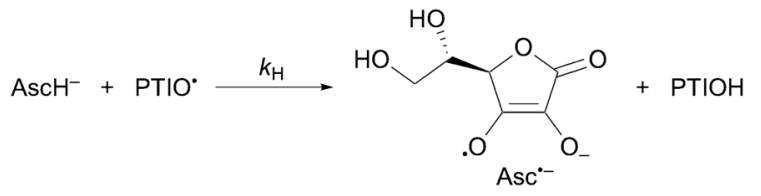

When $\mathrm{H}_{2} \mathrm{O}$ was replaced by $\mathrm{D}_{2} \mathrm{O}$ to prepare the phosphate buffer, the exchangeable $\mathrm{O}-\mathrm{H}$ protons in $\mathrm{AscH}_{2}$ are replaced by deuterons from $\mathrm{D}_{2} \mathrm{O}$ to produce $\mathrm{AscD}_{2}$. The second-order rate constant $\left(k_{\mathrm{D}}\right)$ thus determined for the reaction of $\mathrm{AscD}_{2}$ with $\mathrm{PTIO}^{\bullet}$ was significantly decreased to be $1.9 \times 10^{2} \mathrm{M}^{-1} \mathrm{~s}^{-1}$.

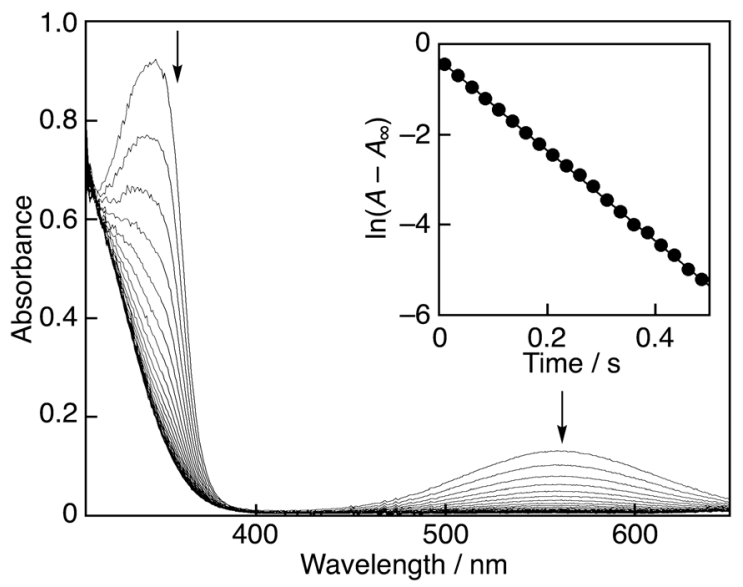

Fig. 2 Spectral change (interval: $25 \mathrm{~ms}$ ) observed during the reaction of $\mathrm{AsCH}_{2}\left(4.0 \times 10^{-3} \mathrm{M}\right)$ with PTIO $\left(9.4 \times 10^{-5} \mathrm{M}\right)$ in phosphate buffer $(0.05 \mathrm{M}, \mathrm{pH} 7.0)$ at $298 \mathrm{~K}$. Inset: The first-order plot of the absorbance at $560 \mathrm{~nm}$.

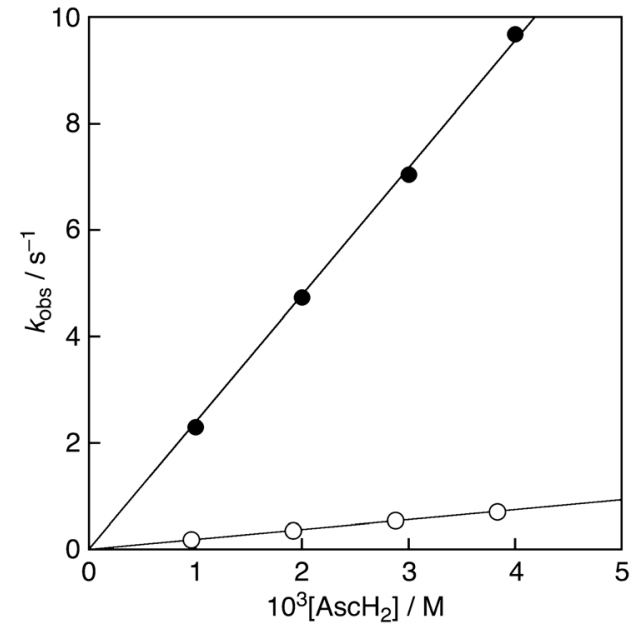

Fig. 3 Plots of pseudo-first-order rate constants $\left(k_{\text {obs }}\right) v s$. concentrations of $\mathrm{AscH}_{2}$ in phosphate buffer $\left(\mathrm{H}_{2} \mathrm{O}, 0.05 \mathrm{M}, \mathrm{pH} 7.0\right)$ (closed circles) and in phosphate buffer $\left(\mathrm{D}_{2} \mathrm{O}, 0.05 \mathrm{M}, \mathrm{pD} 7.0\right.$ ) (open circles).

Thus, the $\mathrm{KIE}\left(k_{\mathrm{H}} / k_{\mathrm{D}}\right)$ is calculated to be 12.8 . Such a large KIE value has clearly precluded an electron-transfer pathway in the oxidation reaction of $\mathrm{AscH}_{2}$ by PTIO ${ }^{\bullet}$. This value is beyond the maximum expected semiclassical value of 7.9 for the dissociation of the $\mathrm{O}-\mathrm{H}$ bond. ${ }^{9}$ We also performed the reaction of $\mathrm{AscH}_{2}$ with $\mathrm{PTIO}^{\bullet}$ in the temperature range from 288 to $308 \mathrm{~K}$ and the $k_{\mathrm{H}}$ and $k_{\mathrm{D}}$ values were determined from the slopes of the linear plots of the $k_{\mathrm{obs}}$ values $v s$. concentrations of $\mathrm{AscH}_{2}$ or $\mathrm{AscD}_{2}$ (Table 1).

Furthermore, as the Arrhenius plots are shown in Fig. 4, linear correlations of $\ln k_{\mathrm{H}}$ vs. $T^{-1}$ and $\ln k_{\mathrm{D}} v s . T^{-1}$ were observed in the reaction of $\mathrm{AscH}_{2}$ with PTIO in the whole temperature range. From the intercepts of Fig. 4, the isotopic ratio of the Arrhenius prefactor $\left(A_{\mathrm{H}} / A_{\mathrm{D}}=0.86\right)$ was obtained. This value can be fitted within the semiclassical limits of 0.7-1.4 for the $A_{\mathrm{H}} / A_{\mathrm{D}}$ value in a hydrogen-transfer process. ${ }^{9}$ The isotopic difference in the activation energies $E_{\mathrm{a}}(\mathrm{D})-E_{\mathrm{a}}(\mathrm{H})$ was $6.8 \mathrm{~kJ} \mathrm{~mol}^{-1}$, which is beyond the difference in zero-point energies of $5.1 \mathrm{~kJ} \mathrm{~mol}^{-1}$. ${ }^{9}$ A large $A_{\mathrm{H}} / A_{\mathrm{D}}$ value ( $\gg 1$ ) in hydrogen transfer of some enzymes has been reported by Klinman et al., proposing a full tunneling mode to explain such observation. ${ }^{3}$ Furthermore, Uršić et al. claimed that quantum mechanical tunneling plays a role in the reaction between $\mathrm{AscH}_{2}$ and TEMPO in water-dioxane $(1: 1 \mathrm{v} / \mathrm{v})$ based on the large KIE value of 31.1 and $E_{\mathrm{a}}(\mathrm{D})-E_{\mathrm{a}}(\mathrm{H})$ value of $8.2 \mathrm{~kJ} \mathrm{~mol}^{-1}$, although the $A_{\mathrm{H}} / A_{\mathrm{D}}$ value is $1.2 .^{5}$ Thus, quantum mechanical tunneling may

Table $1 k_{H}, k_{\mathrm{D}}$ and $k_{\mathrm{H}} / k_{\mathrm{D}}$ values for the reaction of $\mathrm{AsCH}_{2}$ or $\mathrm{AsCD}_{2}$ in phosphate buffer solutions (0.05 M, pH 7.0 or pD 7.0)

\begin{tabular}{llll}
\hline$T / \mathrm{K}$ & $k_{\mathrm{H}} / \mathrm{M}^{-1} \mathrm{~s}^{-1}$ & $k_{\mathrm{D}} / \mathrm{M}^{-1} \mathrm{~s}^{-1}$ & $k_{\mathrm{H}} / k_{\mathrm{D}}$ \\
\hline 288 & $1.5 \times 10^{3}$ & $9.6 \times 10$ & 15.6 \\
293 & $2.0 \times 10^{3}$ & $1.5 \times 10^{2}$ & 13.3 \\
298 & $2.4 \times 10^{3}$ & $1.9 \times 10^{2}$ & 12.8 \\
300 & $2.8 \times 10^{3}$ & $1.9 \times 10^{2}$ & 14.8 \\
303 & $2.9 \times 10^{3}$ & $2.2 \times 10^{2}$ & 13.3 \\
308 & $3.1 \times 10^{3}$ & $2.6 \times 10^{2}$ & 12.2
\end{tabular}




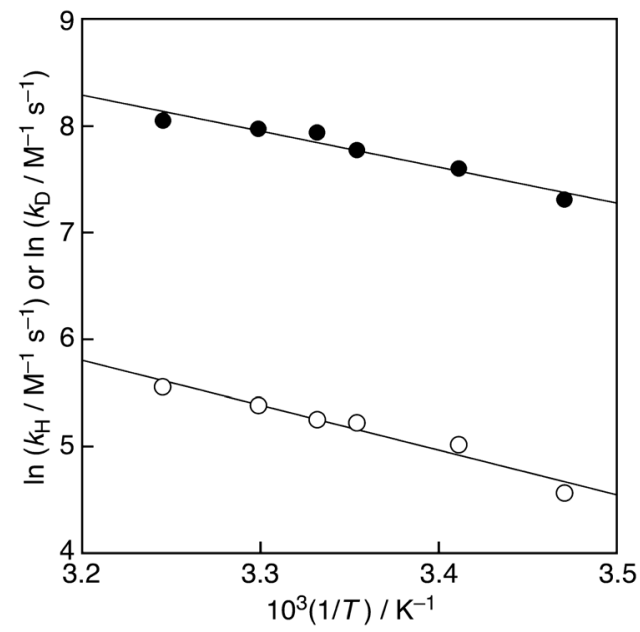

Fig. 4 Arrhenius plots of $\ln k_{H} v s . T^{-1}$ (closed circles) and $\ln k_{\mathrm{D}} v s . T^{-1}$ (open circles) in phosphate buffer $\left(\mathrm{H}_{2} \mathrm{O}, 0.05 \mathrm{M}, \mathrm{pH} 7.0\right)$ and in phosphate buffer $\left(\mathrm{D}_{2} \mathrm{O}, 0.05 \mathrm{M}, \mathrm{pD} 7.0\right)$, respectively.

partly play a role in the reaction between $\mathrm{AscH}_{2}$ and $\mathrm{PTIO}^{\bullet}$ in a phosphate buffer solution.

In summary, a large KIE was observed for the hydrogentransfer reaction from $\mathrm{AscH}_{2}$ to $\mathrm{PTIO}^{\bullet}$. The temperature dependence of the KIE suggests that quantum mechanical tunneling may partly play a role in the reaction, although the isotopic ratio of the Arrhenius prefactor $\left(A_{\mathrm{H}} / A_{\mathrm{D}}\right)$ is within the semiclassical limits. Because there are only a few reports about a large KIE in a reaction involving $\mathrm{AscH}_{2}$, this study provides valuable information for the biological redox reactions including ascorbic acid.

This work was partially supported by Grant-in-Aid (No. 18K06620 to I. N., 20H02779, 20H04819, 18H04650, $17 \mathrm{H} 03010$ and $16 \mathrm{H} 02268$ to K. O.) from the Ministry of Education, Culture, Sports, Science and Technology, Japan. We also thank Dr Hiroaki Kotani (University of Tsukuba) for valuable discussions.

\section{Conflicts of interest}

There are no conflicts to declare.

\section{Notes and references}

1 J. Meisner and J. Kästner, Angew. Chem., Int. Ed., 2016, 55, 5400. 2 P. R. Schreiner, J. Am. Chem. Soc., 2017, 139, 15276.

3 (a) J. P. Klinman and A. R. Offenbacher, Acc. Chem. Res., 2018, 51, 1966; (b) Z. D. Nagel and J. P. Klinman, Nat. Chem. Biol., 2009, 5,543 .

4 J. McFadden and J. Al-Khalili, Proc. R. Soc. A, 2018, 474, 20180674.

5 (a) I. Sajenko, V. Pilepić, C. Jakobušić Brala and S. Uršić, J. Phys. Chem. A, 2010, 114, 3423; (b) A. Karković Marković, C. Jakobušić Brala, V. Pilepić and S. Uršić, Molecules, 2020, 25, 1443.

6 X. Li, J. Agric. Food Chem., 2017, 65, 6288.

7 (a) C. Creutz, Inorg. Chem., 1981, 20, 4449; (b) N. H. Williams and J. K. Yandell, Aust. J. Chem., 1982, 35, 1133.

8 (a) I. Nakanishi, K. Ohkubo, K. Imai, M. Kamibayashi, Y. Yoshihashi, K. Matsumoto, K. Fukuhara, K. Terada, S. Itoh, T. Ozawa and S. Fukuzumi, Chem. Commun., 2015, 51, 8311; (b) I. Nakanishi, K. Ohkubo, M. Kamibayashi, Y. Ogawa, T. Ozawa, K. Matsumoto and S. Fukuzumi, ChemistrySelect, 2016, 1, 3367.

9 R. P. Bell, The Tunnel Effect in Chemistry, Chapman \& Hall, London, 1980. 